Curr Opin Infect Dis. 2010 October ; 23(5): 470-474. doi:10.1097/QCO.0b013e32833da1eb.

\title{
Bacteroides spp. and diarrhea
}

\author{
Elizabeth C. Wick and Cynthia L. Sears \\ Departments of Surgery and Medicine, Johns Hopkins University School of Medicine, Baltimore, \\ Maryland, USA
}

\begin{abstract}
Purpose of review-The purpose of this review is to describe recent progress in the understanding of the role of Bacteroides spp. in human diarrheal diseases and newer murine studies implicating certain Bacteroides spp. in colorectal cancer.
\end{abstract}

Recent findings-Bacteroides fragilis is the only strain of Bacteroides spp. associated with diarrheal disease. Toxin-producing strains of $B$. fragilis, termed enterotoxigenic Bacteroides fragilis (ETBF), are an established cause of diarrheal disease in people. The clinical syndrome associated with ETBF diarrheal disease encompasses abdominal pain, tenesmus and inflammatory diarrhea. Two new studies conducted in mice have further defined the chronic inflammatory response associated with ETBF infection and observed that in the multiple intestinal neoplasia mouse strain, heterozygotes for the adenomatous polyposis coli gene, ETBF infection enhances development of colonic tumors. Separate murine studies have begun to define the role of nontoxin-producing $B$. fragilis as a symbiont, serving possibly to protect the host from colonic inflammation.

Summary-B. fragilis remains the leading anaerobe in human disease. ETBF is emerging as an important cause of human diarrheal disease but additional epidemiologic studies are needed to better understand the role of ETBF human disease.

\section{Keywords}

Bacteroides; diarrhea; enterotoxigenic Bacteroides fragilis

\section{Introduction}

The colon is now known to be one of the most densely populated sites in the human body possessing up to $10^{13}$ bacteria/g stool [1]. This complex community of bacteria predominantly belongs to two major anaerobic bacterial phyla, Bacteroidetes and Firmicutes. Within the Bacteroidetes phylum, Bacteroides spp. comprise about $30 \%$ of the total cultivated flora with greater than 20 established species, which are given below [2].

1. B. acidifaciens

2. B. caccae

3. B. coprocola

4. B. coprosuis

(C) 2010 Wolters Kluwer Health | Lippincott Williams \& Wilkins

Correspondence to Cynthia L. Sears, MD, CRB2 Bldg, Suite \#1M.05, 1550 Orleans Street, Baltimore, MD, 21231, USA, Tel: +1 410 614 0141; fax: +1 410614 8173; csears@jhmi.edu.

The authors have no conflict of interests. 
5. B dorei

6. B. eggerthii

7. B. finegoldii

8. B. fragilis

9. B. goldsteinii

10. B. helcogenes

11. B. intestinalis

12. B. johnsonii

13. B. massiliensis

14. B. nordii

15. B. ovatus

16. B. plebeius

17. B. pyogenes

18. B. salyersai

19. B. thetaiotaomicron

20. B. uniformis

21. B. vulgatus

Bacteroides spp. are usually symbionts that are believed to contribute to the host's nutritional status as well as mucosal and systemic immunity [3•]. In contrast, Bacteroides spp. also contribute to human disease when colonic integrity is disrupted allowing escape into the sterile peritoneum where they act as opportunistic pathogens. Among the Bacteroides spp., the capacity to cause harm is greatest for $B$. fragilis sensu stricto, which remains the leading anaerobe in intra-abdominal abscesses and bloodstream infections with mortality reported as high as $20 \%$ [4-6]. An emerging aspect of $B$. fragilis pathogenicity is the ability of select strains termed enterotoxigenic Bacteroides fragilis (ETBF) to cause diarrheal disease in animals, children, and adults [7•]. Among Bacteroides spp., only ETBF has been associated with diarrheal disease to date. Conversely, nonenterotoxigenic $B$. fragilis (NTBF) strains are proposed to be a possible probiotic with the potential to quell colonic inflammation (Fig. 1). This review will describe how these two molecular subgroups of $B$. fragilis are currently thought to influence colonic health and disease.

\section{The 'good' B. fragilis: nonenterotoxigenic $B$. fragilis}

To date, only two NTBF strains have been sequenced that, along with older data, provide insight into their putative roles as host symbionts and opportunistic pathogens [8,9]. A portion of the genome of NTBF is devoted to facilitating metabolism of a wide array of different polysaccharides found in the colon. The most striking aspect of the NTBF genome is the genes encoding the polysaccharide capsule. NTBF expresses eight distinct polysaccharides [termed polysaccharide A (PSA) to PSH [10]] representing the most complex polysaccharide system known among bacteria. Expression of different polysaccharide capsules facilitates adherence to the colonic mucosa and also, when the organism is outside of the gastrointestinal tract, contributes to virulence and abscess formation. The majority of our understanding of the biology of NTBF is based on studies of a single strain (NCTC 9343), one of the fully sequenced strains. 
Recently, new concepts have emerged about how NTBF may contribute to colonic and human health. Germ-free mice have defects in lymphoid and CD4 ${ }^{+} \mathrm{T}$-cell development. Colonization of germ-free mice with NTBF or treatment of the mice with PSA alone corrects these defects in the mucosal and systemic immune system, restoring the balance between T-helper cell (Th) 1 and Th2 immunity $[11,12]$. Further, in certain murine models of experimental colitis (considered models of inflammatory bowel disease) based on Helicobacter hepaticus colonization or administration of the compound trinitrobenzene sulfonic acid (TNBS), administration of NTBF or PSA alone protects against the development of colitis [11]. These data provided the first demonstration that a single molecule of a common human symbiont could promote beneficial mucosal and, possibly, systemic immune responses that deter inflammatory responses. Additional data suggest that other Bacteroides spp, such as B. thetaiotamicron, contribute essential metabolic functions converting poorly utilized dietary carbohydrates into readily absorbed nutrients [3•]. Thus, the presence of Bacteroides spp. in the colonic flora appears to be integral to human health.

\section{The 'bad' $B$. fragilis: enterotoxigenic $B$. fragilis}

No ETBF strains have yet to be fully sequenced. Available data suggest that these strains differ from NTBF through acquisition of at least one conjugative transposon containing a pathogenicity island or islet with a distinct virulence gene encoding a $20-\mathrm{kDa}$ metalloprotease toxin called the $B$. fragilis toxin or BFT (also known as fragilysin) [7•]. BFT is secreted by ETBF both in vivo (detectable in stool) and in vitro. Three subtypes of BFT (BFT-1, BFT-2 and BFT-3) have been identified to date with BFT-1 expressed by about two-thirds of ETBF isolated around the globe followed by BFT-2 (25\%) and BFT-3 $(\sim 10 \%$ and mostly identified in southeast Asia). Limited biologic differences have been identified to date among these BFT isotypes. BFT stimulates the cleavage of intercellular adhesion protein E-cadherin on colonic epithelial cells, resulting in increased human colon permeability and activates nuclear factor-kappa B signalling, resulting in proinflammatory cytokine secretion by colonic epithelial cells.

In the last year, two reviews have provided a comprehensive summary of ETBF and their clinical expression as well as the pathogenesis of these infections $[7 \bullet, 13]$. Below are the summarized key features of ETBF disease in humans. ETBF were first identified and associated with lamb diarrheal disease in 1984 and with human diarrheal disease in a controlled study reported in 1992 [14,15]. Studies conducted around the globe show that ETBF are consistently associated with human diarrheal disease with the majority of the studies conducted in young children $(<5$ years of age). Children less than 1 year of age are asymptomatically colonized with ETBF, although diarrheal disease due to ETBF emerges after age 1 .

The largest study of ETBF disease in adults was conducted in Sweden [16]. The investigators assessed ETBF in adults hospitalized with acute diarrheal disease compared with isolation of ETBF from healthy outpatient control adults. ETBF was identified in 27\% of 728 patients with diarrhea and $12 \%$ of 194 healthy controls $(P<0.01)$. In this study, $19 \%$ of adults of below 30 years of age, $10.6 \%$ of adults between ages 30 and 60 , and $3.7 \%$ of adults older than 60 years were asymptomatically colonized with ETBF. Among adults between 15 and 30 years of age, ETBF fecal carriage rates were similar for those with and without diarrhea, whereas association of ETBF with diarrheal disease emerged in older patients. In an effort to further define the clinical picture of ETBF infections including intestinal inflammatory responses and the systemic and intestinal antibody responses during ETBF diarrhea, an observational study of children and adults with acute diarrheal illnesses was conducted in Dhaka, Bangladesh [17]. ETBF was identified to cause a clinical syndrome with marked abdominal pain and nonfebrile inflammatory diarrhea in both 
children (age > 1 year) and adults. Fecal leukocytes, lactoferrin, and proinflammatory cytokines [interleukin (IL)-8, tumor necrosis factor-a] as well as systemic and fecal antiBFT responses (IgA, IgG) increased rapidly in ETBF-infected patients. Recently ETBF has also been identified as a potential cause of traveler's diarrhea [18]. US and European travelers visiting Mexico, Guatemala, and India who developed acute diarrheal disease were examined for the prevalence of conventional bacterial enteric pathogens, enterotoxigenic and enteroaggregative Escherichia coli (ETEC, EggEC, respectively) as well as Arcobacter spp. and ETBF in stool samples. One or more pathogens were identified in $84 \%$ of study participants. ETEC was identified in 152 patients (76\% of those examined), with 16 (8\%) individuals positive for $A$. buzleri and 15 (7\%) positive for ETBF in stool samples. Approximately, $50 \%$ of those positive for ETBF were also ETEC positive. The prevalence of ETBF diarrhea varied by location being most common in travelers to Goa, India [6 patients (13\%)].

Studies examining specifically for ETBF in other clinical entities, such as nosocomial or antibiotic-associated diarrhea, are limited and inconclusive. Three small clinical studies have reported a link between ETBF infection and active inflammatory bowel disease as well as colorectal cancer, raising the concern that chronic ETBF colonization may lead to adverse consequences for the host [19-21]. Larger studies utilizing sensitive and specific ETBF diagnostic tools are needed to further assess these preliminary findings.

Studies reported in the last year conducted in newly developed murine models of ETBF disease have added substantively to our understanding of the pathogenesis of ETBF infections and, notably, have demonstrated that, in mice, ETBF is an oncogenic enteric pathogen. In the most commonly used laboratory mouse (C57BL/6 mouse strain), ETBF induces not only acute, symptomatic colitis but also persistent subclinical colonic inflammation and hyperplasia that is present throughout the life span of the mouse [22]. In contrast, in germ-free mice, ETBF colitis was lethal suggesting that the conventional mouse flora dampens ETBF pathology. Colonic histopathology demonstrated mucosal thickening with inflammatory cell infiltration, crypt abscesses, and epithelial cell exfoliation, erosion, and ulceration. In contrast, NTBF (strain NCTC 9343) caused no histopathology. The detection of chronic colonic inflammation associated with asymptomatic ETBF colonization combined with in-vitro studies showing that BFT activates pro-oncogenic cellular signaling led to the hypothesis that ETBF is an oncogenic bacterium. This hypothesis was recently tested in the multiple intestinal neoplasia (Min) mouse strain, heterozygotes for the adenomatous polyposis coli (APC) gene, which develop intestinal tumors when the second copy of the $A P C$ gene spontaneously mutates [22]. The Min mouse is the murine homolog of the familial adenomatosis polyposis (FAP) syndrome that inexorably leads to colon cancer in those affected. Min mice mostly develop small bowel tumors beginning at approximately 3 months, and by $4-6$ months, have developed usually less than five colon tumors per mouse. In contrast, Min mice colonized with ETBF develop not only chronic, asymptomatic colitis but also intraepithelial colon tumor foci detected by 1 week after colonization. Neither shaminoculated nor Min mice colonized with NTBF develop excess colon tumors. By 1-2 months after ETBF inoculation, ETBF colonization is associated with a marked increase in gross colonic tumor formation not observed in controls (Fig. 2). Tumor induction and numbers correlate with microscopic colon hyperplasia and inflammation and occur predominantly in the distal colon, consistent with the distribution of human colorectal cancer. Small bowel tumors did not increase in ETBF-colonized Min mice, consistent with the known colonic niche for $B$. fragilis.

ETBF further rapidly induces infiltration of the colonic lamina propria with IL-17-producing $\mathrm{CD} 3^{+} \mathrm{CD} 4^{+} \mathrm{T}$-cells and $\mathrm{CD} 3^{+} \mathrm{CD} 4^{-} \gamma \delta$-T-cells. Tumor formation was significantly inhibited by administration of blocking antibody to IL-17, thus confirming the role of IL-17 in 
tumorigenesis in this murine model of colorectal neoplasia. These studies show that a common human colonic bacterium induces inflammation-induced colon neoplasia, in part, through a Th17-dependent immune pathway. Extension of these observations demonstrating ETBF-induced colitis and tumorigenesis in murine models to humans will require detailed study of the epidemiology of ETBF infection and colorectal pathology.

\section{Other links between Bacteroides spp. and diarrheal disease}

Additional observations suggest that Bacteroides spp. may also contribute to exerting a protective effect in colitis and diarrheal disease. Clostridium difficile diarrhea is associated with a shift in the composition of the commensal flora and, specifically, diminished colonization with Bacteroides spp has been reported in $C$. difficile diarrhea and antibioticassociated diarrhea [24]. It has long been hypothesized that restoration of a balance in the colonic microbiota may be an effective treatment for recurrent $C$. difficile infection. Fecal transplantation in a patient with chronic $C$. difficile was conducted in an effort to restore the Bacteroides spp. [25]. Prior to transplantation, the patient's flora was deficient in Bacteroides spp. with Firmicutes dominating. By 2 weeks after transplantation, the patient's flora resembled the donor's flora and was mainly Bacteroides spp.; the chronic diarrhea resolved. Bacteroides spp. have also been implicated in being protective against chemotherapy-induced diarrhea. Under normal conditions, the microbiota is embedded within the protective mucous membrane overlying the intestinal epithelial cells. Chemotherapeutic agents, including 5-fluoruracil (5-FU) destroy the epithelial lining of the gastrointestinal tract and can be associated with severe colitis and diarrhea. A recent study examined the effect of 5-FU-induced mucin destruction on the colonic flora in mice [26•]. 5Fluoruracil treatment, in addition to causing colitis, was associated with fewer fecal Lactobacilli and Bacteroides spp. and increased E. coli, suggesting that shifts in the flora may contribute to chemotherapy-induced gastrointestinal toxicity. A similar murine study focusing on irinotecan-associated diarrhea found that after administration of irinotecan (a topoisomerase inhibitor), the flora was dominated by E. coli, Staphylococcus and Clostridium spp. whereas Lactobacillus, Bifidobacterium and Bacteroides spp. had been depleted [27]. These studies are consistent with the data suggesting a role for NTBF in colonic health but do not define which Bacteroides spp. or even if Bacteroides spp, rather than other members of the microbiota, are crucial to the associations show.

\section{Conclusions}

The Bacteroides spp. are a complex group of organisms. Among this group of organisms, only $B$. fragilis and $B$. thetaiotamicron have been studied in any depth experimentally and clinically. $B$. fragilis has been and remains the leading anaerobe in human disease. Clinical and experimental data have distinguished two major molecular $B$. fragilis subgroups NTBF and ETBF, with only ETBF being associated with diarrheal disease in children and adults. Newer experimental studies suggest that NTBF may be crucial to colonic mucosal integrity and immune development whereas long-term ETBF colonization may induce chronic inflammation with the potential for oncogenic transformation. Human epidemiologic studies are needed to better understand the role of $B$. fragilis and other Bacteroides spp. in human health and disease.

\section{References and recommended reading}

Papers of particular interest, published within the annual period of review, have been highlighted as:

- of special interest 
$\bullet$ of outstanding interest

Additional references related to this topic can also be found in the Current World Literature section in this issue (p. 535).

1. Costello EK, Lauber CL, Hamady M, et al. Bacterial community variation in human body habitats across space and time. Science. 2009; 326:1694-1697. [PubMed: 19892944]

2. Wexler HM. Bacteroides: the good, the bad, and the nitty-gritty. Clin Microbiol Rev. 2007; 20:593621. [PubMed: 17934076]

3. Neish AS. Microbes in gastrointestinal health and disease. Gastroenterology. 2009; 136:65-80. [PubMed: 19026645] This is a comprehensive review of the role of the microbiome in promoting health and disease.

4. Redondo MC, Arbo MD, Grindlinger J, Snydman DR. Attributable mortality of bacteremia associated with the Bacteroides fragilis group. Clin Infect Dis. 1995; 20:1492-1496. [PubMed: 7548498]

5. Polk BF, Kasper DL. Bacteroides fragilis subspecies in clinical isolates. Ann Intern Med. 1977; 86:569-571. [PubMed: 322563]

6. Lassmann B, Gustafson DR, Wood CM, Rosenblatt JE. Reemergence of anaerobic bacteremia. Clin Infect Dis. 2007; 44:895-900. [PubMed: 17342637]

7. Sears CL. Enterotoxigenic Bacteroides fragilis: a rogue among symbiotes. Clin Microbiol Rev. 2009; 22:349-369. [PubMed: 19366918] This is a comprehensive review outlining progress in understanding the physiology and pathogenesis of ETBF infection.

8. Kuwahara T, Yamashita A, Hirakawa H, et al. Genomic analysis of Bacteroides fragilis reveals extensive DNA inversions regulating cell surface adaptation. Proc Natl Acad Sci U S A. 2004; 101:14919-14924. [PubMed: 15466707]

9. Cerdeno-Tarraga AM, Patrick S, Crossman LC, et al. Extensive DNA inversions in the B. fragilis genome control variable gene expression. Science. 2005; 307:1463-1465. [PubMed: 15746427]

10. Coyne MJ, Chatzidaki-Livanis M, Paoletti LC, Comstock LE. Role of glycan synthesis in colonization of the mammalian gut by the bacterial symbiont Bacteroides fragilis. Proc Natl Acad Sci U S A. 2008; 105:13099-13104. [PubMed: 18723678]

11. Mazmanian SK, Round JL, Kasper DL. A microbial symbiosis factor prevents intestinal inflammatory disease. Nature. 2008; 453:620-625. [PubMed: 18509436]

12. Mazmanian SK, Liu CH, Tzianabos AO, Kasper DL. An immunomodulatory molecule of symbiotic bacteria directs maturation of the host immune system. Cell. 2005; 122:107-118. [PubMed: 16009137]

13. Holton J. Enterotoxigenic Bacteroides fragilis. Curr Infect Dis Rep. 2008; 10:99-104. [PubMed: 18462582]

14. Sack RB, Myers LL, Almeido-Hill J, et al. Enterotoxigenic Bacteroides fragilis: epidemiologic studies of its role as a human diarrhoeal pathogen. J Diarrhoeal Dis Res. 1992; 10:4-9. [PubMed: 1619243]

15. Myers LL, Firehammer BD, Shoop DS, Border MM. Bacteroides fragilis: a possible cause of acute diarrheal disease in newborn lambs. Infect Immun. 1984; 44:241-244. [PubMed: 6538870]

16. Zhang G, Svenungsson B, Karnell A, Weintraub A. Prevalence of enterotoxigenic Bacteroides fragilis in adult patients with diarrhea and healthy controls. Clin Infect Dis. 1999; 29:590-594. [PubMed: 10530453]

17. Sears CL, Islam S, Saha A, et al. Association of enterotoxigenic Bacteroides fragilis infection with inflammatory diarrhea. Clin Infect Dis. 2008; 47:797-803. [PubMed: 18680416]

18. Jiang ZD, Dupont HL, Brown EL, et al. Microbial etiology of travelers' diarrhea in Mexico, Guatemala, and India: importance of enterotoxigenic Bacteroides fragilis and Arcobacter species. $\mathrm{J}$ Clin Microbiol. 2010; 48:1417-1419. [PubMed: 20107088]

19. Toprak NU, Yagci A, Gulluoglu BM, et al. A possible role of Bacteroides fragilis enterotoxin in the aetiology of colorectal cancer. Clin Microbiol Infect. 2006; 12:782-786. [PubMed: 16842574] 
20. Basset C, Holton J, Bazeos A, et al. Are Helicobacter species and enterotoxigenic Bacteroides fragilis involved in inflammatory bowel disease? Dig Dis Sci. 2004; 49:1425-1432. [PubMed: 15481314]

21. Prindiville TP, Sheikh RA, Cohen SH, et al. Bacteroides fragilis enterotoxin gene sequences in patients with inflammatory bowel disease. Emerg Infect Dis. 2000; 6:171-174. [PubMed: 10756151]

22. Rhee KJ, Wu S, Wu X, et al. Induction of persistent colitis by a human commensal, enterotoxigenic Bacteroides fragilis, in wild-type C57BL/6 mice. Infect Immun. 2009; 77:17081718. [PubMed: 19188353]

23. Wu S, Rhee KJ, Albesiano E, et al. A human colonic commensal promotes colon tumorigenesis via activation of T helper type $17 \mathrm{~T}$ cell responses. Nat Med. 2009; 15:1016-1022. [PubMed: 19701202] This is the first report of a human commensal promoting colon tumorigenesis. Future translational studies are required to understand the role of ETBF in human colorectal cancer.

24. Chang JY, Antonopoulos DA, Kalra A, et al. Decreased diversity of the fecal microbiome in recurrent Clostridium difficile-associated diarrhea. J Infect Dis. 2008; 197:435-438. [PubMed: 18199029]

25. Khoruts A, Dicksved J, Jansson JK, Sadowsky MJ. Changes in the composition of the human fecal microbiome after bacteriotherapy for recurrent Clostridium difficile-associated diarrhea. J Clin Gastroenterol. 2010; 44:354-360. [PubMed: 20048681]

26. Stringer AM, Gibson RJ, Logan RM, et al. Gastrointestinal microflora and mucins may play a critical role in the development of 5-fluorouracil-induced gastrointestinal mucositis. Exp Biol Med (Maywood). 2009; 234:430-441. [PubMed: 19176868] This study by Stringer et al. begins to demonstrate the beneficial effects of $B$. fragilis colonization with respect to preventing diarrheal disease.

27. Stringer AM, Gibson RJ, Logan RM, et al. Faecal microflora and beta-glucuronidase expression are altered in an irinotecan-induced diarrhea model in rats. Cancer Biol Ther. 2008; 7:1919-1925. [PubMed: 18927500] 


\section{Bacteroides fragilis}

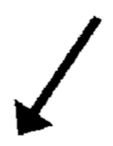

Nontoxigenic bacteroides fragilis

NTBF

Promotes mucosal health

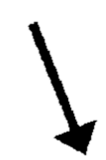

Enterotoxigenic bacteroides fragilis

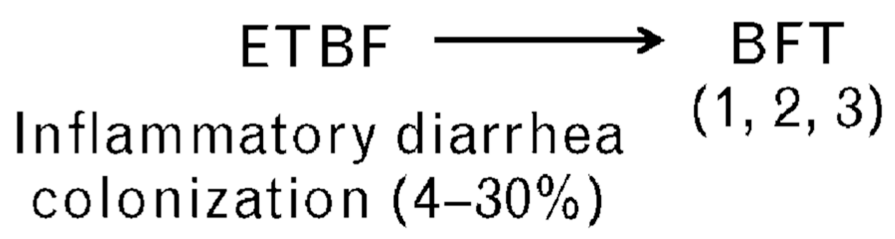

Figure 1. Molecular subtypes of $B$. fragilis sensu stricto

$B$. fragilis commonly colonize the colons of humans. Two molecular subtypes of $B$. fragilis are now identified termed as NTBF and ETBF. ETBF are distinguished by secretion of the BFT. Three distinct isotypes of BFT exist but, to date, a single ETBF strain secretes only one isotype of BFT. Murine data suggest that NTBF promote colon mucosal health (see text) and human data indicate that ETBF induce inflammatory diarrhea but also are frequent clinically silent colon colonizers. BFT, Bacteroides fragilis toxin; ETBF, enterotoxigenic Bacteroides fragilis; NTBF, nonenterotoxigenic Bacteroides fragilis. 


\section{Two months}

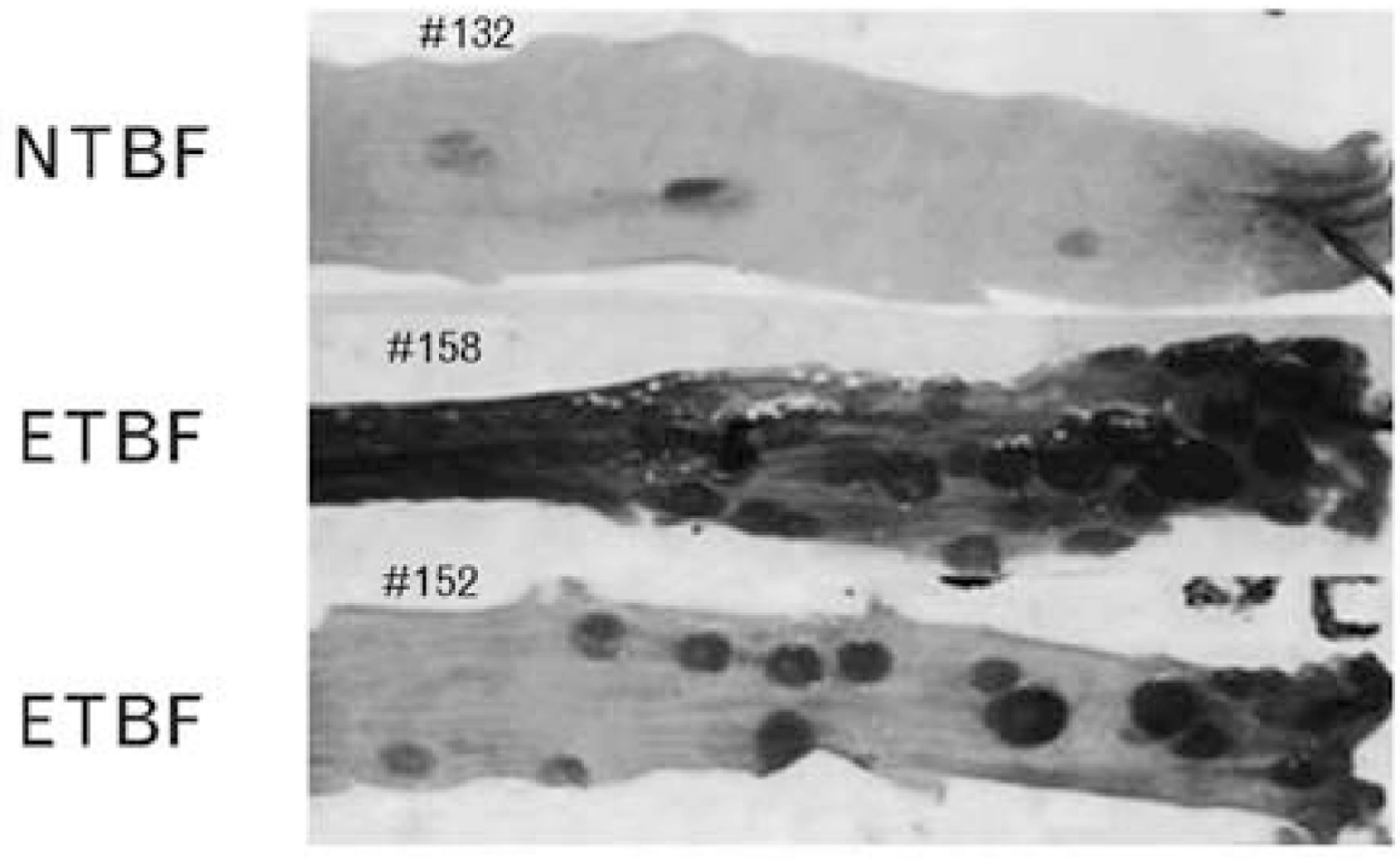

Figure 2. Enterotoxigenic Bacteroides fragilis induce colon tumors in multiple intestinal neoplasia mice

The photograph shows the distal colons of Min mice colonized for 2 months with either NTBF or ETBF. Only ETBF-colonized Min mice display excess gross tumors in colons stained with methylene blue. ETBF, enterotoxigenic Bacteroides fragilis; Min, multiple intestinal neoplasia; NTBF, nonenterotoxigenic Bactersoides fragilis. Reproduced with permission from $[23 \bullet]$. 